Review

\title{
Perfringolysin O: The Underrated Clostridium perfringens Toxin?
}

\author{
Stefanie Verherstraeten 1, Evy Goossens 1, Bonnie Valgaeren 2, Bart Pardon 2, \\ Leen Timbermont ${ }^{1}$, Freddy Haesebrouck ${ }^{1}$, Richard Ducatelle ${ }^{1}$, Piet Deprez ${ }^{2}$, \\ Kristin R. Wade ${ }^{3}$, Rodney Tweten ${ }^{3}$ and Filip Van Immerseel ${ }^{1, *}$
}

1 Department of Pathology, Bacteriology and Avian Diseases, Faculty of Veterinary Medicine, Ghent University, Salisburylaan 133, 9820 Merelbeke, Belgium;

E-Mails: s.verherstraeten@gmail.com (S.V.); evy.goossens@ugent.be (E.G.); leen.timbermont@ugent.be (L.T.); freddy.haesebrouck@ugent.be (F.H.); richard.ducatelle@ugent.be (R.D.)

2 Department of Internal Medicine and Clinical Biology of Large Animals, Faculty of Veterinary Medicine, Ghent University, Salisburylaan 133, 9820 Merelbeke, Belgium; E-Mails: bonnie.valgaeren@ugent.be (B.V.); bart.pardon@ugent.be (B.P.); piet.deprez@ugent.be (P.D.)

3 Department of Microbiology and Immunology, University of Oklahoma Health Sciences Center, Oklahoma City, OK 73104, USA; E-Mails: kristin-wade@ouhsc.edu (K.R.W.); rodney-tweten@ouhsc.edu (R.T.)

* Author to whom correspondence should be addressed; E-Mail: filip.vanimmerseel@ugent.be; Tel.: +32-9264-7748; Fax: +32-9264-7789.

Academic Editor: Michel R. Popoff

Received: 9 April 2015 / Accepted: 8 May 2015 / Published: 14 May 2015

Abstract: The anaerobic bacterium Clostridium perfringens expresses multiple toxins that promote disease development in both humans and animals. One such toxin is perfringolysin $\mathrm{O}$ (PFO, classically referred to as $\theta$ toxin), a pore-forming cholesterol-dependent cytolysin (CDC). PFO is secreted as a water-soluble monomer that recognizes and binds membranes via cholesterol. Membrane-bound monomers undergo structural changes that culminate in the formation of an oligomerized prepore complex on the membrane surface. The prepore then undergoes conversion into the bilayer-spanning pore measuring approximately 250-300 $\AA$ in diameter. PFO is expressed in nearly all identified $C$. perfringens strains and harbors interesting traits that suggest a potential undefined role for PFO in disease development. Research has demonstrated a role for PFO in gas gangrene progression and bovine 
necrohemorrhagic enteritis, but there is limited data available to determine if PFO also functions in additional disease presentations caused by $C$. perfringens. This review summarizes the known structural and functional characteristics of PFO, while highlighting recent insights into the potential contributions of PFO to disease pathogenesis.

Keywords: cholesterol-dependent cytolysin; gas gangrene; myonecrosis; necrohemorrhagic enteritis; enterotoxaemia; calves

\section{Introduction}

Clostridium perfringens is an anaerobic, spore-forming Gram-positive bacterium often found as a normal inhabitant of animal and human intestines [1-3]. However, by mechanisms and stimuli that are not fully understood, $C$. perfringens undergoes rapid proliferation, while producing several toxins, resulting in disease onset. Classification of $C$. perfringens strains is based on the production of $\alpha, \beta, \varepsilon$ and $\mathrm{l}$ toxins, considered the four major clostridial toxins. Additional toxins are also expressed and secreted by $C$. perfringens, such as the pore-forming toxin perfringolysin $\mathrm{O}$ (PFO), formerly referred to as $\theta$ toxin [4,5]. PFO, in synergy with $\alpha$ toxin, is involved in the development of gas gangrene and necrohemorrhagic enteritis in calves [6,7]. PFO might also be important in other diseases caused by C. perfringens, since PFO is known to potentiate the lethal effect of $\varepsilon$ toxin in a mouse model for type D enterotoxaemia, a naturally occurring disease in sheep and goats [8]. The PFO pore-forming mechanism has been well studied, leading to several insights into the PFO structure and function. However, the role of PFO in disease development is not often evaluated in pathogenesis models, despite the fact that PFO harbors characteristics that suggest a more important role in animal and human diseases than previously thought. We present herein an overview of the known characteristics of PFO and hypothesize about PFO contributions to the pathology of animal and human diseases.

PFO belongs to the cholesterol-dependent cytolysin (CDC) family, which acts as pore-forming toxins on cholesterol-containing membranes [9]. Similar toxins have been identified in Streptococcus, Bacillus, Listeria and many other genera. These CDCs share a high degree of primary structural homology. PFO is viewed as the archetype CDC, and thus, data presented in this review for PFO can be partially extrapolated to other CDCs and provide the basis for a general CDC pore-forming mechanism [10].

\section{Genetics}

The genome of Clostridium perfringens consists of a single circular chromosome and additional extra-chromosomal plasmids. Many of the toxins produced are plasmid-encoded, including $\beta, \varepsilon$ and 1 toxins, while the genes encoding PFO $(p f o A)$ and $\alpha$ toxin $(p l c)$ are located on the chromosome [11-18]. The $p f o A$ gene is suspected to be encoded by nearly all $C$. perfringens strains, although genome comparisons revealed that the majority of the enterotoxin-producing food poisoning strains lack pfo $A[16,17,19,20]$. The structural gene $p f o A$ has been cloned, sequenced and mapped [21-23]. The primary protein structure derived from the nucleotide sequence includes 500 amino acid residues and a 27-residue signal peptide [24]. Based on these data, Tweten [24] predicted a molecular weight of 
52,469 daltons (Da) for PFO. However, variations occur in the primary structure and in the PFO chromosomal location $[19,24,25]$. Recombination presumably explains the variations in the location and sequence of $p f o A$ and other chromosome-encoded virulence genes, although the primary structure of PFO is well conserved $[11,25,26]$. The most conserved region of $p f o A$ surrounds the undecapeptide, a tryptophan-rich loop that contains three tryptophan residues and the only cysteine residue in secreted PFO [10,24].

\section{PFO Structure}

PFO contains a typical signal peptide that facilitates its secretion by the general secretory pathway (GSP), which results in an extracellular water-soluble monomer [10,24]. This signal peptide is recognized by the GSP and is cleaved upon passage through the cell membrane [27,28]. Solovyova et al. [29] hypothesized that PFO forms dimers in solution at high concentrations, and the crystals of PFO exhibited a head-to-tail dimer [30]. Whether PFO forms dimers at physiological concentration remains unclear. The solved crystal structure reveals that PFO monomers have an elongated structure divided into four domains that are dominated by $\beta$-strands (Figure 1) [30]. Domain 4 (D4) contains two $\beta$-sheets, each consisting of four $\beta$-strands (D4 $\beta 1-4$ and D4 $\beta 5-8$ ) packed together in a $\beta$-sandwich structure connected by four loops (L1, L2, L3 and undecapeptide) (Figures 1 and 2) [30,31]. Domain 3 (D3) consists of one core $\beta$-sheet (D3 $\beta 1-5$ ) flanked by two sets of three $\alpha$-helices (D3 $\alpha 1-3$ and D3 $\alpha 4-6$ ) (Figure 1; see also Figure 4a) [30]. An additional $\alpha$-helix ( $\alpha 7$ ) connects $\beta 5$ with domain 1 (D1). Domain 1 and 2 (D2) connect D3 and D4 (Figure 1). The elongated D2 contains a $\beta$-sheet, whereas D1 consists of a $\beta$-sheet and four $\alpha$-helices (Figure 1) [30].

In the membrane-embedded pore complex, the $\beta$-strands of D3 from several monomers form a single closed cylindrical $\beta$-sheet, forming a $\beta$-barrel. The concerted action of at least 35 monomers is required to create a large transmembrane $\beta$-barrel of $250-300 \AA$ in diameter [30,32-34].

\section{Membrane Binding}

A conserved feature of CDCs is the requirement for cholesterol, a common constituent of mammalian membranes, for their cytolytic activity [9,35]. Therefore, cholesterol was long suspected to be the receptor for the CDCs, because cholesterol is essential for pore formation, and the activity of CDCs is inhibited when CDCs are pre-exposed to cholesterol [35-40]. Results on intermedilysin (ILY), a CDC produced by Streptococcus intermedius, indicate that not all CDCs utilize cholesterol for binding [41,42]. Nevertheless, cholesterol remains crucial for pore formation in these CDCs. 


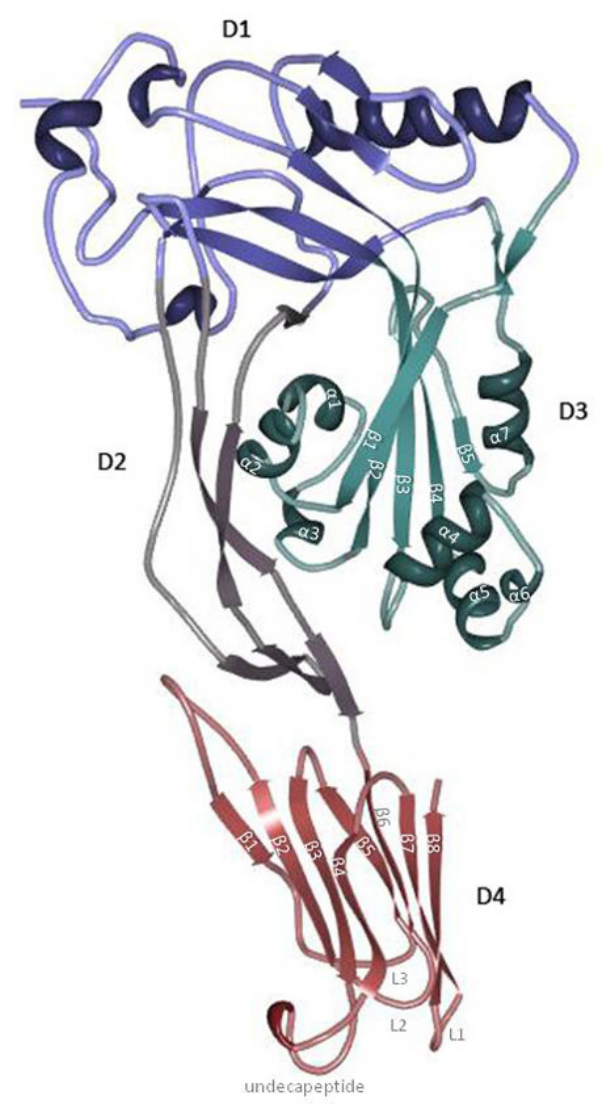

Figure 1. Perfringolysin O's (PFO) structure. PFO is dominated by $\beta$-strands and is divided into four domains. Domain 4 (red; D4) consists of two $\beta$-sheets of four $\beta$-strands (D4 $\beta 1-4$ and D4 $\beta 5-8$ ) packed together in a $\beta$-sandwich structure connected by four loops (L1, L2, L3 and undecapeptide). Domain 3 (green; D3) contains one core $\beta$-sheet (D3 $\beta 1-5$ ) flanked by two sets of three $\alpha$-helices (D3 $\alpha 1-3$ and D3 $\alpha 4-6$ ) and an additional $\alpha$-helix ( $\alpha 7$ ) that connects $\beta 5$ with domain 1 (blue; D1). Domain 1 and domain 2 (purple; D2) connect D3 and D4. D2 is elongated and contains a $\beta$-sheet. D1 consists of a $\beta$-sheet and four $\alpha$-helices. The figure was made with RCSB PDB Protein Workshop 4.1.0 (RCSB Protein Data Bank, Piscataway, NJ and La Jolla, CA, USA, 2014) and adapted with Adobe Photoshop CS3 extended (Adobe Systems Incorporated, San Jose, CA, USA, 2007).

The cytolytic mechanism of PFO begins with D4-mediated binding to the plasma membrane of the eukaryotic cell $[9,31,33,43]$. D4 interacts with the membrane via loops at the tip of the domain that connect the two $\beta$-sheets (Figure 2). The rest of D4 does not contact the membrane surface [31,33]. One of these loops corresponds to the conserved undecapeptide (Figure 2). Until relatively recently, it was thought that this tryptophan-rich loop functioned as the cholesterol binding motif, since modification of this region influenced PFO binding and pore-forming activity [44-47]. Furthermore, the cysteine in the undecapeptide seemed to be involved in the cytolytic mechanism, since mutation and oxidation of this cysteine also altered the conformation of the tryptophan-rich loop and hindered membrane binding (Figure 2) [48]. However, more recent evidence shows that the three other loops, L1-L3, are important in cholesterol recognition and binding rather than the undecapeptide (Figure 2) [47]. The undecapeptide, on the other hand, contributes to both the anchoring of PFO to the membrane and to the allosteric coupling of binding to distal conformational and structural changes necessary for the insertion of the 
$\beta$-barrel in the membrane, which explains why mutations of these tryptophans influence the cytotoxic activity $[47,49,50]$. In addition, cysteine modification prevents the prepore to pore transition (molecular mechanism of pore formation below) by influencing the tryptophan-rich loop [47]. The cholesterol binding motif was subsequently identified by Farrand et al. [51], who showed that only two residues, threonine-490 and leucine-491 in L1, are crucial in the recognition and binding of cholesterol in the membrane (Figure 2). The Thr-Leu pair is collectively termed the cholesterol-recognition motif, or CRM. Following binding initiation by the CRM, the L2 and L3 of D4 insert into the membrane and stabilize binding, thereby orientating PFO perpendicular to the membrane $[31,34,52]$.

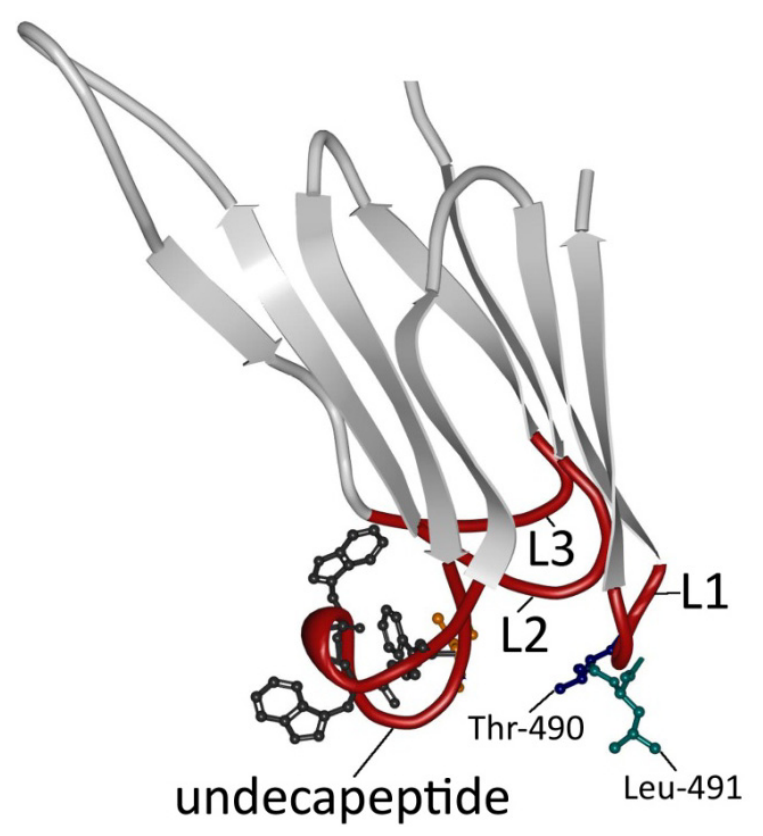

Figure 2. Detailed view of PFO domain 4. Domain 4 (D4) consists of two $\beta$-sheets of four $\beta$-strands (light grey; D4 $\beta 1-4$ and D4 $\beta 5-8$ ) connected by four loops (red; L1, L2, L3 and undecapeptide). The undecapeptide contains three out of six tryptophans (dark grey) in PFO and the only cysteine (orange) present in the secreted form. Recent results have shown that only the threonine (blue) and the leucine (green) in L1 are essential for the recognition and binding of the membrane [49]. The figure was made with RCSB PDB Protein Workshop 4.1.0 (RCSB Protein Data Bank, Piscataway, NJ and La Jolla, CA, USA, 2014) and adapted with Adobe Photoshop CS3 extended (Adobe Systems Incorporated, San Jose, CA, USA, 2007).

Interestingly, the CRM motif is conserved in all known CDCs, even in intermedilysin (ILY), which utilizes the human GPI-anchored CD59 (huCD59) as a membrane binding receptor, rather than cholesterol [30,31,43]. Following the ILY binding interaction with huCD59, ILY uses the CRM motif and L2 and L3 in exactly the same manner as PFO to form a cholesterol-dependent contact. ILY oligomerizes into the prepore intermediate, while bound to huCD59, but disengages from huCD59 during conversion to the pore. Thus, ILY cholesterol binding serves to anchor the ILY pore to the membrane following huCD59 disengagement $[51,53]$.

Because PFO only binds to membranes that contain a substantial amount of cholesterol, it was proposed that the binding was associated with the sphingolipid- and cholesterol-rich liquid ordered membrane domains or lipid rafts (Figure 3) [43,54-57]. This hypothesis was contradicted by Nelson et al. [58] and 
Flanagan et al. [36], who did not find an association between the presence of membrane rafts and the ability of PFO to bind. However, studies have demonstrated that the binding capacity of PFO depends on the accessibility of the cholesterol, which is influenced by packing of the phospholipid headgroups and the saturation of the cholesterol-phospholipid acyl chain, both of which can affect how tightly they pack with cholesterol $[36,55,58]$. More recent research showed that PFO has some affinity for lipid rafts, but the association is highest and strongest at the borders of the liquid ordered and liquid disordered membrane domains, where cholesterol seems more accessible to PFO (Figure 3) [59]. The binding of PFO is therefore not merely controlled by the amount of cholesterol present, but also by the lipid environment of the cholesterol. The thickness of the membrane bilayer may also affect the efficiency of pore formation, as modifying the length of the TMH can hinder its insertion into bilayers of various widths [60]. This is not altogether surprising, as the TMH length in the wild-type toxin should be optimal for TMH insertion into the targeted lipid environment surrounding membrane cholesterol.

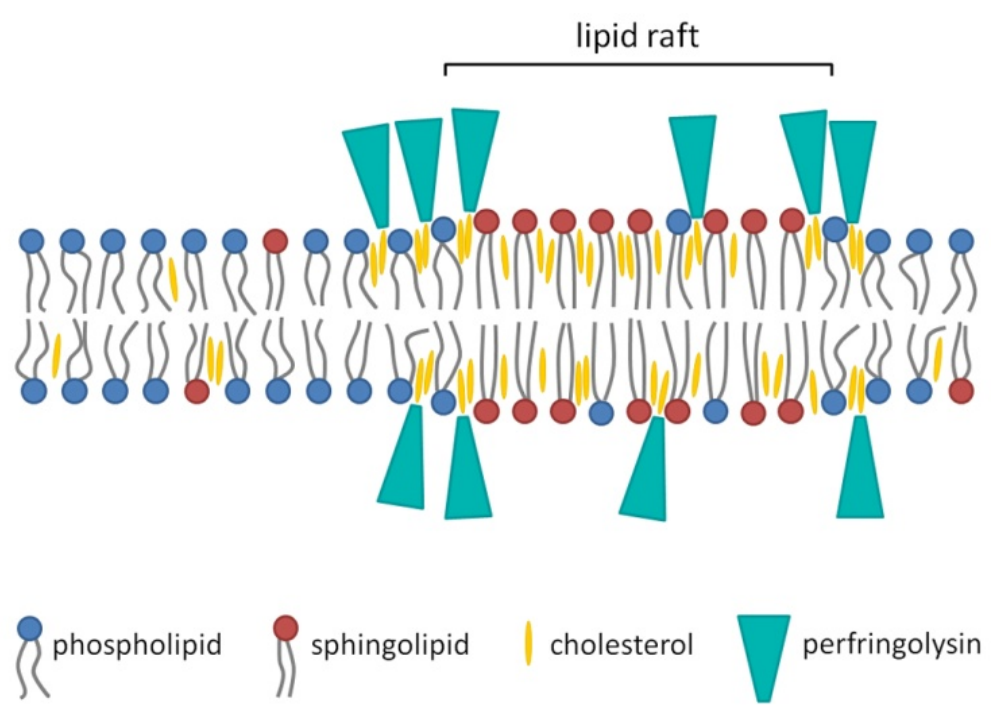

Figure 3. Schematic diagram of the association of PFO with the membrane bilayer. The binding of PFO to the membrane is not merely associated with sphingolipid- and cholesterol-rich liquid ordered membrane domains or lipid rafts. The association is highest and strongest on the edges of the ordered membrane domain, where cholesterol seems more accessible for PFO [59]. The figure was made with Microsoft Office PowerPoint 2007 (Microsoft Corporation, Redmond, WA, USA, 2007).

\section{Molecular Mechanism of Pore Formation}

Within the D3 core $\beta$-sheet, $\beta$-strand 4 ( $\beta 4$ ) forms four hydrogen bonds with $\beta$-strand $5(\beta 5)$, which prevents the association of $\beta 4$ with $\beta$-strand 1 ( $\beta 1$ ) from an adjacent monomer (Figure $4 \mathrm{a}$ ). The $\beta 4-\beta 5$ interaction prevents the spontaneous oligomerization of soluble monomers [9,61]. The binding of D4 to the membrane is coupled to conformational changes in D3, causing $\beta 5$ to rotate around a conserved glycine-glycine pivot away from the core $\beta$-sheet (Figure $4 \mathrm{~b}$ ). As a result, $\beta 4$ becomes exposed and can associate with the always-exposed $\beta 1$ in the core $\beta$-sheet from an adjacent monomer (Figures 4c and 5c) [61-63]. 
a. D3 in soluble monomer

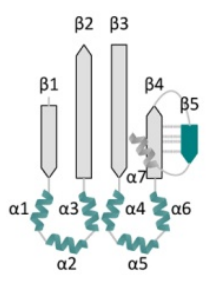

b. D3 in membranebound monomer

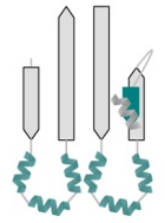

c. D3 in prepore complex

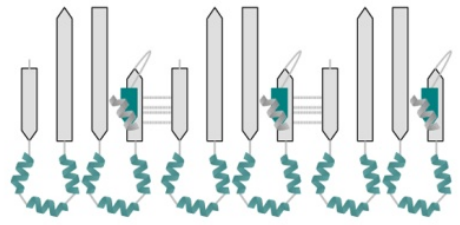

d. D3 in pore complex

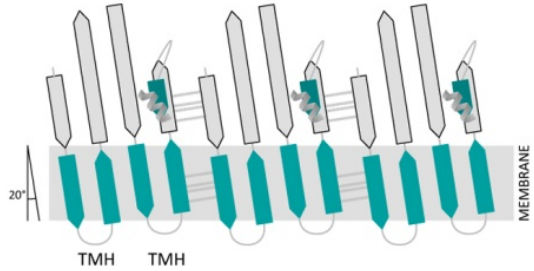

Figure 4. Overview of the conformational changes of domain 3 in the transition from a water-soluble monomer to the pore complex. Domain 3 (D3) contains a $\beta$-sheet (D3 $\beta 1-5$ ) flanked by two sets of three $\alpha$-helices (D3 $\alpha 1-3$ and $\alpha 4-6$ ) and an additional $\alpha$-helix $(\alpha 7)$ that connects $\beta 5$ with domain 1 (D1). In the water-soluble monomer, $\beta 4$ forms hydrogen bonds with $\beta$-strand $\beta 5$ ((a) D3 in the soluble monomer). D4 binding to the membrane elicits conformational changes in D3, which causes the rotation of $\beta 5$ away from the core $\beta$-sheet, thus exposing the edge of $\beta 4$ ((b) D3 in the membrane-bound monomer). As a result, $\beta 4$ can form contacts with an always-exposed $\beta 1$ in the core $\beta$-sheet of an adjacent monomer ((c) D3 in the prepore complex). Upon conversion of the prepore to the pore, the two $\alpha$-helical bundles refold into two amphipathic transmembrane $\beta$-hairpins (TMHs) ((d) D3 in the pore complex). In the pore complex, the $\beta$-strands of D3 monomers are tilted 20 degrees perpendicular to the membrane with a right-hand twist. The figure was made with Graphisoft ArchiCAD 13 (Graphisoft, Budapest, Central Hungary, Hungary, 2009).

In the water-soluble monomer, the core $\beta$-sheet of D3 is flanked by two sets of $\alpha$-helices (D3 $\alpha 1-3$ and D3 $\alpha 4-6$ ), which minimize the exposure of the hydrophobic side chains (Figure 4a) [31,64,65]. As a result of D4 binding to the membrane, conformational changes in D3 are induced that promote the formation of the prepore structure $[61,63]$. The prepore complex is composed of oligomerized monomers that have yet to insert their $\beta$-barrel (Figures 4c, 5c) [66]. After formation of the prepore, the two $\alpha$-helices unfurl into two amphipathic transmembrane $\beta$-hairpins (TMH1 and TMH2), which insert into the membrane in the pore complex (Figures $4 d, 5 d$ ) [64,65,67]. At first, these $\beta$-hairpins extend from the core $\beta$-sheet and move dynamically $[33,52,62,68]$. In the pore complex, the TMHs form backbone hydrogen bonds with TMHs of adjacent molecules to form the contiguous $\beta$-barrel (Figures $4 \mathrm{~d}, 5 \mathrm{~d}$ ). All monomers contribute two TMHs that are inserted into the membrane bilayer in a concerted, synchronized fashion to form the $\beta$-barrel $[62,66,68]$. TMH-synchronized insertion is energetically more favorable than the domino-type insertion of TMHs from individual monomers [69,70]. The TMHs completely span the membrane, with the hydrophobic surfaces facing the lipid core of the membrane and the hydrophilic surfaces exposed to the aqueous pore $[65,67]$.

In the pore complex, the individual $\beta$-strands of $\mathrm{D} 3$ are tilted $20^{\circ}$ perpendicular to the membrane with a right-hand twist (Figure 4d) [62]. A rotation of the D3 core $\beta$-sheet relative to D4 is needed to untwist the core $\beta$-sheet and align the TMHs (Figure $5 \mathrm{~d}$ ). D3 does not directly contact D4, but forms an interface with both D1 and D2 that connects D3 with D4 (Figures 1, 5b) [30,31,43]. The stability of D1 increases upon membrane binding, which may be important for disengagement of the D2,3 interface [71]. When D1 is destabilized by the substitution of a tryptophan residue (Trp-165 in PFO), the interface between D2,3 
fails to disengage [63,71]. Additionally, Wade et al. [72] recently showed that the rotation of $\beta 5$ away from the core $\beta$-sheet in D3 allows the formation of a strong intermolecular electrostatic interaction, which drives the transition from the prepore to the pore by providing the free energy necessary to disrupt the interface between D3 and D1,2. These studies highlight the importance of the domain stability and the interactions between intra- and inter-domains for pore formation.

Czajkowsky et al. [34] and Ramachandran et al. [52] showed that D1 and D3 both undergo a 35-40 A vertical collapse towards the membrane, suggesting that a conformational change must occur in D2 to facilitate this collapse. Such a conformational change is consistent with the bulge on the outer surface of the membrane-inserted oligomeric complex shown in a 3D reconstruction of the pneumolysin (PLY) pore complex, a CDC produced by Streptococcus pneumoniae [73]. Additionally, crystallographic modeling predicts that D4 undergoes a rotation relative to D2 that breaks several contacts between D2 and D3, thereby allowing the $\alpha$-helical bundle to unfold and then refold into TMH (Figure 5d) [64]. Figure 5 summarizes the sequence of the general mechanism of pore complex formation by PFO.
a. soluble monomer
b. membrane- bound monomer
c. prepore complex
d. pore complex
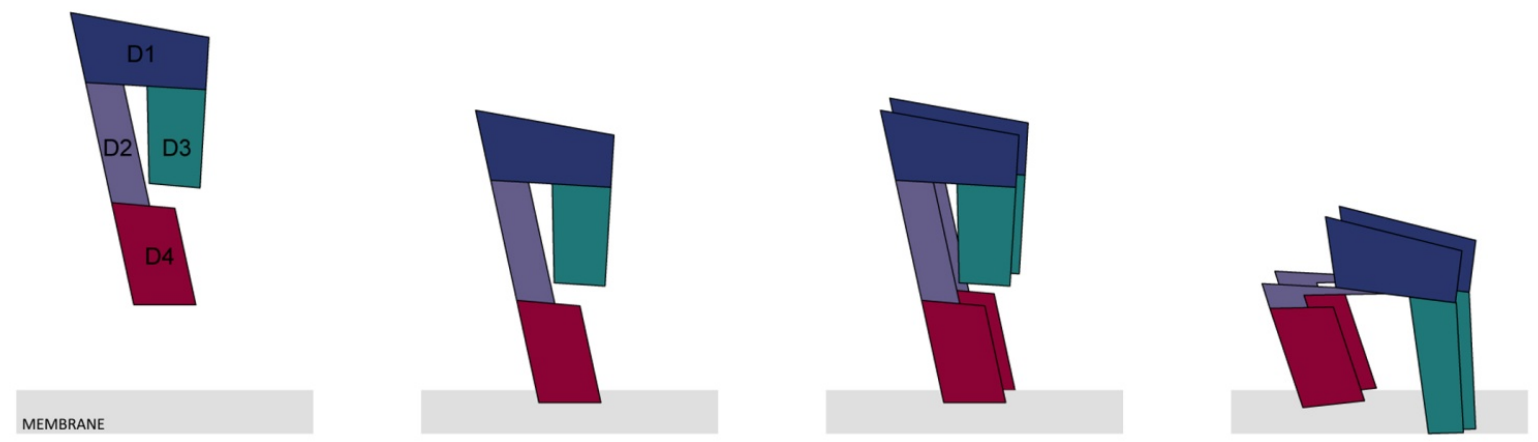

Figure 5. Overview of the general mechanism of the transition from a water-soluble monomer to the pore complex. PFO is secreted as a water-soluble monomer ((a) water-soluble monomer). Upon encountering a cholesterol-containing membrane, D4 (red) interacts with the membrane at the tip of the domain ((b) membrane-bound monomer). This triggers a conformational change in domain 3 (green; D3); thereby, D3 of adjacent monomers can interact with each other, and this causes the formation of a prepore complex ((c) prepore complex). The prepore complex is inserted into the membrane bilayer ((d) pore complex). A collapse of the PFO molecule occurs, which moves domains 3 and 1 (blue; D1) closer to the membrane. This is caused by a rotation of D4 that triggers the bending of domain 2 (purple; D2). The latter reduces the strength of the binding between D3 and D2, and thereby, D3 and D1 can move towards the membrane. The figure was made with Graphisoft ArchiCAD 13 (Graphisoft, Budapest, Central Hungary, Hungary, 2009).

\section{Genetic Regulation}

A quorum-sensing system homologous to the accessory gene regulator system (agr) of Staphylococcus aureus was found in C. perfringens, which could regulate the expression of several virulence factors in response to external stimuli [74-76]. Ohtani et al. [71] and Vidal et al. [76] showed that the expression of PFO and $\alpha$ toxin are regulated by the agr system, as the mutation of the agr locus 
influenced the early log-phase expression of both toxins. C. perfringens also harbors an additional quorum-sensing system homologous to the luxS system [77]. A luxS mutant has a reduced expression of PFO at the mid-exponential growth phase [77]. Additionally, the two-component signal transduction system consisting of the VirS sensor protein and the gene response regulator VirR regulates the expression of PFO, $\alpha$ toxin, collagenase and several housekeeping genes [78-81]. It is not yet completely clear if the agr and luxS system are coupled to the VirR/VirS system or by which stimuli the VirR/VirS system is activated. The signaling molecules and the mode of action remain to be elucidated $[75,76,82]$.

The activation of VirS causes phosphorylation of VirR, allowing VirR to bind two VirR boxes, i.e., 12-bp repeated sequences located upstream of the pfo gene encoding PFO, which causes a direct regulation of $p f o A$ transcription [75,80,83-85]. The expression of the PFO structural gene $p f o A$ is abolished after mutation of VirR or VirS [79-81,84]. Shimizu et al. [22] suspected that the upstream $p f o R$ gene coded for a regulatory protein involved in the expression of the PFO structural gene. Awad and Rood [86] contradicted this by showing that mutating $p f o R$ did not influence $p f o A$ expression. They hypothesized that under certain experimental conditions, the $p f o R$ gene could have a regulatory role, such as in an infected lesion, but this has not been demonstrated.

\section{The Role of PFO in Disease}

Clostridium perfringens is the most important causative agent of clostridial myonecrosis (also called gas gangrene) $[6,87]$. This disease can arise when the anaerobic bacterium is introduced into muscle tissue, often as a consequence of a traumatic injury [88-90]. Clostridial myonecrosis is reported in several animal species, such as dogs, cats, cattle, sheep, goats and horses, but also occurs in humans [90]. The disease is characterized by rapid spreading of tissue necrosis within the muscle (thus the term myonecrosis), which can lead to death caused by systemic toxemia and shock, with very high mortality rates, if not promptly treated $[88,89]$. It is widely accepted that $\alpha$ toxin is an essential causative toxin involved in gas gangrene, because only strains producing $\alpha$ toxin are able to induce the typical pathology of myonecrosis with thrombosis in a mouse myonecrosis model [91-93]. The role of PFO in gas gangrene is less well understood. It appears to work synergistically with $\alpha$ toxin to effect peripheral to myonecrosis leukostasis and intravascular coagulopathy, whereas the majority of the myonecrosis can be attributed to $\alpha$ toxin alone $[6,18,93-96]$. Decreased mortality was observed after immunization with PFO prior to intramuscular injection of $C$. perfringens in mice [95,97]. Awad et al. [6] then demonstrated that PFO acts in synergy with $\alpha$ toxin to cause gas gangrene, as virulence of a mutant lacking both genes was drastically decreased in a mouse myonecrosis model. Furthermore, complementation with both toxins could produce a more severe pathology than the strain complemented with $\alpha$ toxin alone. These results clearly suggest that PFO and $\alpha$ toxin are involved in the pathology of clostridial myonecrosis or gas gangrene. However, the individual contributions of both toxins to disease are not fully defined.

Vascular leukostasis and the paucity of leukocyte infiltration to the site of infection are two characteristics of gas gangrene [6,93]. Intramuscular injection of a crude clostridial toxin preparation triggers the formation of intravascular aggregates consisting of platelets, which initially move freely within blood vessels [93,98]. Fibrin and leukocytes are rapidly added to the aggregate, thus trapping the aggregates and obstructing the blood vessels. The thrombi reduce or even completely stop the local blood flow $[98,99]$. This process impairs the movement of inflammatory cells to the infected tissue, which could 
explain the lack of phagocytic cells at the later stages of a gas gangrene infection. $\alpha$ toxin is shown to directly activate gpIIbIIIa, a receptor on platelets involved in platelet aggregation [100-102]. PFO causes an augmented expression of adhesion molecules, such as CD11b/CD18 on leukocytes and intracellular adhesion molecule 1 (ICAM-1) and platelet-activating factor on human endothelial cells [94,97,103-105]. The increased expression of platelet-activating factor is linked to leukocyte adhesion to human endothelial cells after exposure to $\alpha$ toxin $[99,102,106]$. Therefore, the increased expression of the platelet and endothelial cell adhesion factors, as well as chemokines might also contribute to the adhesion of platelet-leukocyte aggregates to endothelial cells lining the blood vessel, which contributes to thrombosis and subsequent reduction in blood flow [100,102].

This strong leukocyte adhesion to endothelial cells also impairs the transendothelial migration of leukocytes and subsequent infiltration of the infection site [94,97,102,107]. This is of benefit for $C$. perfringens residing in the tissue, as this simultaneously promotes the anaerobic conditions of the tissue and reduces inflammation [18]. Moreover, a direct cytotoxic effect of PFO and $\alpha$ toxin on endothelial cells has been demonstrated, which likely also impairs leukocyte transmigration $[99,107]$. This disruption of the endothelium also contributes to progressive edema [107]. Furthermore, the lack of acute inflammatory cells in the infected tissue could also be due to a direct suppression of the immune response by PFO and $\alpha$ toxin, as a direct cytolytic effect on leukocytes has been observed in the presence of a high concentration of PFO or $\alpha$ toxin [108].

While the later stages of a myonecrosis infection are characterized by a paucity of phagocytic cells, the presence of phagocytic cells could be of importance to control the bacteria at the onset of infection, when there are few bacteria present in the tissue. C. perfringens is able to survive in the presence of macrophages and can escape the phagosome [109]. Successful phagosome escape is only accomplished in the presence of both $\alpha$ toxin and PFO. Furthermore, PFO is the most important factor for macrophage cytotoxicity [110].

In summary, PFO causes macrophage cytotoxicity in the early stages of myonecrosis and is important for thrombi formation in the later stages of infection. The latter is caused by effects on the expression of adhesion factors and chemokines by endothelial cells and leukocytes. Although PFO is involved, $\alpha$ toxin is clearly also involved, suggesting these two toxins act synergistically.

Uzal et al. [18] suggested that PFO is not a main causative toxin for most intestinal diseases caused by $C$. perfringens, as type $\mathrm{C}$ strains lacking the $p f o A$ and $\alpha$ toxin gene caused lesions comparable to natural cases in a rabbit ileal loop model, and the $p f o A$ gene is absent in the genome of most enterotoxin-producing food poisoning strains [16,17,19,20,111]. Recently, however, the involvement of PFO in the pathogenesis of bovine necrohemorrhagic enteritis or enterotoxaemia has been demonstrated [7]. This disease is most typically characterized by sudden death with macroscopic post-mortem identification of necrotic and hemorrhagic lesions in the small intestine [4,112]. Necrohemorrhagic enteritis is a major cause of mortality in veal calves, causing important economic losses [113-115]. Comparable to the data on myonecrosis, PFO acts synergistically with $\alpha$ toxin in the development of this disease, since a mutant lacking both genes had a decreased ability to induce necrohemorrhagic lesions and only complementation with both genes restored activity to wild-type levels [7].

While gas gangrene is characterized by tissue necrosis, thrombosis formation and a lack of leukocyte infiltration at the site of infection, bovine necrohemorrhagic enteritis is associated with capillary congestion, hemorrhages and inflammation [116-118]. PFO and $\alpha$ toxin are involved in both gas 
gangrene and necrohemorrhagic enteritis, but the different phenotypes suggest that these toxins may act in different ways in the disease pathology. Both toxins have a strong cytotoxic effect on bovine endothelial cells, which might indicate that these toxins act by targeting the endothelium, potentially explaining capillary hemorrhages [7].

Intravenous injection of recombinant PFO in rabbits causes an increase in cardiac output $[95,96]$. This is not due to a direct cardiotoxic effect, but rather to a reduction in vascular resistance and subsequent vasodilatation $[6,95,96]$. Relaxation of the blood vessels is proposed to result as an indirect effect of PFO on endogenous mediators, such as cytokines [95,96]. After injection of recombinant $\alpha$ toxin in rabbits, however, the vascular resistance was maintained while a reduction in cardiac output and arterial pressure were observed $[95,96,99,119]$. This was attributed to a direct effect of $\alpha$ toxin on the myocardial contractility $[95,96]$. Rabbits inoculated intravenously with crude $C$. perfringens supernatant exhibit an initial increase in blood flow, linked to the reduction in vascular resistance due to PFO [96]. However, a reduction in cardiac output and arterial pressure are observed afterwards, which are attributed to $\alpha$ toxin $[96,99,119]$. These results, combined with the pathological observations of bovine necrohemorrhagic enteritis (i.e., inflammation and hemorrhages) and loss of vascular integrity, seem to be in accordance with the role of PFO in this disease. On the contrary, hemorrhages are not observed in myonecrosis. Moreover, gas gangrene does not appear to be associated with a reduction in vascular resistance, as during an amputation of an infected limb/area, removal of all non-bleeding tissue is recommended [98]. Intramuscular injection of a crude clostridial toxin preparation in rodents triggers the formation of intravascular aggregates, leading to thrombi formation [93,98]. No intravascular aggregates and subsequent thrombosis are observed in bovine necrohemorrhagic enteritis, which partially explains the inflammation present in bovine necrohemorrhagic enteritis, which is in contrast to the lack of leukocyte infiltration (and subsequent inflammation) in gas gangrene. These observations indicate that the effects exerted by both PFO and $\alpha$ toxin are species and target organ dependent. In advanced stages of disease, myonecrosis develops further into systemic toxemia and multi-organ failure caused by cardiovascular collapse as a result of the effects on systemic blood pressure and cardiac function linked with $\alpha$ toxin $[87,107]$. Bovine necrohemorrhagic enteritis is characterized by sudden death, which might also be due to a direct effect of $\alpha$ toxin on myocardial contractility. However, more research is needed to explain the observed differences in pathology between gas gangrene and bovine necrohemorrhagic enteritis. Further insights into the mode of action of these toxins are needed to unravel their effects on different target organs and identify the relative contribution of both toxins to pathogenesis.

The possible role of PFO in other diseases caused by $C$. perfringens needs to be more clearly defined. Several clostridial disease phenotypes are thought to result from a specific causative toxin. However, PFO could be of importance in these diseases, as well. For example, recently, Fernandez-Miyakawa et al. [8] demonstrated that PFO potentiated the lethal effect of $\varepsilon$ toxin in a mouse model for type D enterotoxaemia. This disease naturally occurs predominantly in sheep and goats and is characterized by a rapid proliferation of $C$. perfringens type D strains in the intestine, leading to toxemia and death, sometimes preceded by neurological symptoms [15,120]. $\varepsilon$ toxin is generally accepted as the causative toxin for type D enterotoxaemia, since it is the most lethal toxin of Clostridium perfringens, and intravenous inoculation of $\varepsilon$ toxin reproduces lesions comparable to natural cases [15,120-122]. Recently, Garcia et al. [117] confirmed the essential role of $\varepsilon$ toxin in type $\mathrm{D}$ enterotoxaemia, as the mutation of the $\varepsilon$ toxin gene in a virulent type D strain eliminated the lethal effect and clinical signs in an intraduodenal inoculation model 
in mice, sheep and goats. Most type D strains also produce $\alpha$ toxin and PFO. Therefore, Fernandez-Miyakawa et al. [8] evaluated the possible synergistic effect of both on $\varepsilon$ toxin. Intravenous and intragastric injection of $\varepsilon$ toxin in combination with PFO reduced the survival time of mice. For $\alpha$ toxin, a reduction in survival time was only observed after intravenous injection [8]. These results show that the lethal effect of $\varepsilon$ toxin can be enhanced by PFO and $\alpha$ toxin.

\section{Remaining Questions}

Cholesterol is essential for pore formation for all cholesterol-dependent cytolysins. Despite the presence of the cholesterol recognition motif, or CRM, in all known CDCs, the timing and role of cholesterol binding can differ between the different CDCs. For PFO, cholesterol seems to be the membrane binding receptor, since the recognition of cholesterol by the CRM in the membrane is the first step in membrane binding. However, the interaction of ILY with cholesterol only occurs after ILY binding to huCD59, with cholesterol then serving to anchor the ILY pore to the membrane following huCD59 disengagement. Whether non-CD59 restricted CDCs utilize an additional receptor is unclear; one study has implicated glycans as a possible membrane receptor, but the data remain uncertain because of the high concentration of competitor glycan required to inhibit CDC binding to cholesterol [123].

Additionally, it is still not clear if sphingolipid- and cholesterol-rich liquid ordered membrane domains or lipid rafts are more prone to cytolysin binding, since the results of completed studies to date are contradictory and inconclusive.

Some gaps remain in our knowledge of the genetic regulation, as well. As already mentioned, there is no insight yet into the signaling molecules and the mode of action of the VirR/VirS system. In addition, the regulatory role of the upstream $p f o R$ gene still needs to be clarified.

In spite of widespread PFO production among $C$. perfringens strains, the role of PFO has been underinvestigated in disease development. Future research should take into account the potential contribution of PFO in the pathogenesis of C. perfringens-induced diseases, with particular attention on the possible synergistic effect of PFO on the causative toxins. Furthermore, all potentially involved organs should be considered, as the effects can be target organ dependent. Moreover, it is also important that the early events in the disease development are investigated, as PFO might have a significant role in the onset of the disease, whereas its contribution at later stages may be less important.

\section{Acknowledgments}

Stefanie Verherstraeten was supported by a PhD fellowship from the Institute for Science and Technology, Flanders, under Contract Number 090910. Rodney Tweten was supported by a grant from the U.S. National Institutes of Health, under Grant Number AI37657.

\section{Author Contributions}

Stefanie Verherstraeten, Kristin R. Wade, Rodney Tweten and Filip Van Immerseel drafted the manuscript. Stefanie Verherstraeten made the figures. Evy Goossens, Bonnie Valgaeren, Bart Pardon, Leen Timbermont, Freddy Haesebrouck, Richard Ducatelle and Piet Deprez critically revised the manuscript. All authors read and approved the final manuscript. 


\section{Conflicts of Interest}

Sources of financial support have been acknowledged, and the authors declare no conflict of interest.

\section{References}

1. Rood, J.I. Virulence genes of Clostridium perfringens. Annu. Rev. Microbiol. 1998, 52, 333-360.

2. Lebrun, M.; Filee, P.; Mousset, B.; Desmecht, D.; Galleni, M.; Mainil, J.G.; Linden, A. The expression of Clostridium perfringens consensus $\beta 2$ toxin is associated with bovine enterotoxaemia syndrome. Vet. Microbiol. 2007, 120, 151-157.

3. Morris, W.E.; Dunleavy, M.V.; Diodati, J.; Berra, G.; Fernandez-Miyakawa, M.E. Effects of Clostridium perfringens $\alpha$ and $\varepsilon$ toxins in the bovine gut. Anaerobe 2012, 18, 143-147.

4. Songer, J.G. Clostridial enteric diseases of domestic animals. Clin. Microbiol. Rev. 1996, 9, 216-234.

5. Songer, J.G.; Miskimmins, D.W. Clostridium perfringens type E enteritis in calves: Two cases and a brief review of the literature. Anaerobe 2004, 10, 239-242.

6. Awad, M.M.; Ellemor, D.M.; Boyd, R.L.; Emmins, J.J.; Rood, J.I. Synergistic effects of $\alpha$-toxin and perfringolysin $\mathrm{O}$ in Clostridium perfringens-mediated gas gangrene. Infect. Immun. 2001, 69, 7904-7910.

7. Verherstraeten, S.; Goossens, E.; Valgaeren, B.; Pardon, B.; Timbermont, L.; Vermeulen, K.; Schauvliege, S.; Haesebrouck, F.; Ducatelle, R.; Deprez, P.; et al. The synergistic necrohemorrhagic action of Clostridium perfringens perfringolysin and $\alpha$ toxin in the bovine intestine and against bovine endothelial cells. Vet. Res. 2013, 44, 45.

8. Fernandez-Miyakawa, M.E.; Jost, B.H.; Billington, S.J.; Uzal, F.A. Lethal effects of Clostridium perfringens $\varepsilon$ toxin are potentiated by $\alpha$ and perfringolysin-O toxins in a mouse model. Vet. Microbiol. 2008, 127, 379-385.

9. Heuck, A.P.; Savva, C.G.; Holzenburg, A.; Johnson, A.E. Conformational changes that effect oligomerization and initiate pore formation are triggered throughout perfringolysin $\mathrm{O}$ upon binding to cholesterol. J. Biol. Chem. 2007, 282, 22629-22637.

10. Hotze, E.M.; Tweten, R.K. Membrane assembly of the cholesterol-dependent cytolysin pore complex. Biochim. Biophys. Acta 2012, 1818, 1028-1038.

11. Popoff, M.R.; Bouvet, P. Genetic characteristics of toxigenic Clostridia and toxin gene evolution. Toxicon 2013, 75, 63-89.

12. Fisher, D.J.; Fernandez-Miyakawa, M.E.; Sayeed, S.; Poon, R.; Adams, V.; Rood, J.I.; Uzal, F.A.; McClane, B.A. Dissecting the contributions of Clostridium perfringens type $\mathrm{C}$ toxins to lethality in the mouse intravenous injection model. Infect. Immun. 2006, 74, 5200-5210.

13. Marvaud, J.C.; Stiles, B.G.; Chenal, A.; Gillet, D.; Gibert, M.; Smith, L.A.; Popoff, M.R. Clostridium perfringens $\mathrm{t}$ toxin. Mapping of the Ia domain involved in docking with $\mathrm{Ib}$ and cellular internalization. J. Biol. Chem. 2002, 277, 43659-43666.

14. Nagahama, M.; Hayashi, S.; Morimitsu, S.; Sakurai, J. Biological activities and pore formation of Clostridium perfringens $\beta$ toxin in HL 60 cells. J. Biol. Chem. 2003, 278, 36934-36941.

15. Popoff, M.R. $\varepsilon$ toxin: A fascinating pore-forming toxin. FEBS J. 2011, 278, 4602-4615. 
16. Myers, G.S.; Rasko, D.A.; Cheung, J.K.; Ravel, J.; Seshadri, R.; DeBoy, R.T.; Ren, Q.; Varga, J.; Awad, M.M.; Brinkac, L.M.; et al. Skewed genomic variability in strains of the toxigenic bacterial pathogen. Clostridium perfringens. Genome Res. 2006, 16, 1031-1040.

17. Rood, J.I.; Cole, S.T. Molecular genetics and pathogenesis of Clostridium perfringens. Microbiol. Rev. 1991, 55, 621-648.

18. Uzal, F.A.; Freedman, J.C.; Shrestha, A.; Theoret, J.R.; Garcia, J.; Awad, M.M.; Adams, V.; Moore, R.J.; Rood, J.I.; McClane, B.A. Towards an understanding of the role of Clostridium perfringens toxins in human and animal disease. Future Microbiol. 2014, 9, 361-377.

19. Deguchi, A.; Miyamoto, K.; Kuwahara, T.; Miki, Y.; Kaneko, I.; Li, J.; McClane, B.A.; Akimoto, S. Genetic characterization of type A enterotoxigenic Clostridium perfringens strains. PLoS ONE 2009, 4, e5598.

20. Ma, M.; Li, J.; McClane, B.A. Genotypic and phenotypic characterization of Clostridium perfringens isolates from Darmbrand cases in post-World War II Germany. Infect. Immun. 2012, 80, 4354-4363.

21. Tweten, R.K. Cloning and expression in Escherichia coli of the perfringolysin O ( $\theta$-toxin) gene from Clostridium perfringens and characterization of the gene product. Infect. Immun. 1988, 56, 3228-3234.

22. Shimizu, T.; Okabe, A.; Minami, J.; Hayashi, H. An upstream regulatory sequence stimulates expression of the perfringolysin O gene of Clostridium perfringens. Infect. Immun. 1991, 59, 137-142.

23. Canard, B.; Cole, S.T. Genome organization of the anaerobic pathogen Clostridium perfringens. Proc. Natl. Acad. Sci. USA 1989, 86, 6676-6680.

24. Tweten, R.K. Nucleotide sequence of the gene for perfringolysin O ( $\theta$-toxin) from Clostridium perfringens: Significant homology with the genes for streptolysin $\mathrm{O}$ and pneumolysin. Infect. Immun. 1988, 56, 3235-3240.

25. Sawires, Y.S.; Songer, J.G. Clostridium perfringens: Insight into virulence evolution and population structure. Anaerobe 2006, 12, 23-43.

26. Rooney, A.P.; Swezey, J.L.; Friedman, R.; Hecht, D.W.; Maddox, C.W. Analysis of core housekeeping and virulence genes reveals cryptic lineages of Clostridium perfringens that are associated with distinct disease presentations. Genetics 2006, 172, 2081-2092.

27. Sandkvist, M. Type II secretion and pathogenesis. Infect. Immun. 2001, 69, 3523-3535.

28. Lee, V.T.; Schneewind, O. Protein secretion and the pathogenesis of bacterial infections. Genes Dev. 2001, 15, 1725-1752.

29. Solovyova, A.S.; Nollmann, M.; Mitchell, T.J.; Byron, O. The solution structure and oligomerization behavior of two bacterial toxins: Pneumolysin and perfringolysin O. Biophys. J. 2004, 87, 540-552.

30. Rossjohn, J.; Feil, S.C.; McKinstry, W.J.; Tweten, R.K.; Parker, M.W. Structure of a cholesterol-binding, thiol-activated cytolysin and a model of its membrane form. Cell 1997, 89, 685-692.

31. Ramachandran, R.; Heuck, A.P.; Tweten, R.K.; Johnson, A.E. Structural insights into the membrane-anchoring mechanism of a cholesterol-dependent cytolysin. Nat. Struct. Biol. 2002, 9 , $823-827$.

32. Dang, T.X.; Milligan, R.A.; Tweten, R.K.; Wilson-Kubalek, E.M. Helical crystallization on nickel-lipid nanotubes: Perfringolysin O as a model protein. J. Struct. Biol. 2005, 152, 129-139. 
33. Heuck, A.P.; Tweten, R.K.; Johnson, A.E. Assembly and topography of the prepore complex in cholesterol-dependent cytolysins. J. Biol. Chem. 2003, 278, 31218-31225.

34. Czajkowsky, D.M.; Hotze, E.M.; Shao, Z.; Tweten, R.K. Vertical collapse of a cytolysin prepore moves its transmembrane $\beta$-hairpins to the membrane. EMBO J. 2004, 23, 3206-3215.

35. Alouf, J.E.; Geoffroy, C.; Pattus, F.; Verger, R. Surface properties of bacterial sulfhydryl-activated cytolytic toxins. Interaction with monomolecular films of phosphatidylcholine and various sterols. Eur. J. Biochem. 1984, 141, 205-210.

36. Flanagan, J.J.; Tweten, R.K.; Johnson, A.E.; Heuck, A.P. Cholesterol exposure at the membrane surface is necessary and sufficient to trigger perfringolysin $\mathrm{O}$ binding. Biochemistry 2009, 48, 3977-3987.

37. Nollmann, M.; Gilbert, R.; Mitchell, T.; Sferrazza, M.; Byron, O. The role of cholesterol in the activity of pneumolysin, a bacterial protein toxin. Biophys. J. 2004, 86, 3141-3151.

38. Howard, J.G.; Wallace, K.R.; Wright, G.P. The inhibitory effects of cholesterol and related sterols on haemolysis by streptolysin O. Br. J. Exp. Pathol. 1953, 34, 174-180.

39. Mitsui, K.; Saeki, Y.; Hase, J. Effects of cholesterol evulsion on susceptibility to perfringolysin O of human erythrocytes. Biochim. Biophys. Acta 1982, 686, 177-181.

40. Shany, S.; Bernheimer, A.W.; Grushoff, P.S.; Kim, K.S. Evidence for membrane cholesterol as the common binding site for cereolysin, streptolysin O and saponin. Mol. Cell Biochem. 1974, 3, 179-186.

41. Giddings, K.S.; Johnson, A.E.; Tweten, R.K. Redefining cholesterol's role in the mechanism of the cholesterol-dependent cytolysins. Proc. Natl. Acad. Sci. USA 2003, 100, 11315-11320.

42. Giddings, K.S.; Zhao, J.; Sims, P.J.; Tweten, R.K. Human CD59 is a receptor for the cholesterol-dependent cytolysin intermedilysin. Nat. Struct. Mol. Biol. 2004, 11, 1173-1178.

43. Heuck, A.P.; Hotze, E.M.; Tweten, R.K.; Johnson, A.E. Mechanism of membrane insertion of a multimeric $\beta$-barrel protein: Perfringolysin $O$ creates a pore using ordered and coupled conformational changes. Mol. Cell 2000, 6, 1233-1242.

44. Sekino-Suzuki, N.; Nakamura, M.; Mitsui, K.I.; Ohno-Iwashita, Y. Contribution of individual tryptophan residues to the structure and activity of $\theta$-toxin (perfringolysin $\mathrm{O}$ ), a cholesterol-binding cytolysin. Eur. J. Biochem. 1996, 241, 941-947.

45. Nakamura, M.; Sekino, N.; Iwamoto, M.; Ohno-Iwashita, Y. Interaction of $\theta$-toxin (perfringolysin O), a cholesterol-binding cytolysin, with liposomal membranes: Change in the aromatic side chains upon binding and insertion. Biochemistry 1995, 34, 6513-6520.

46. Iwamoto, M.; Ohno-Iwashita, Y.; Ando, S. Role of the essential thiol group in the thiol-activated cytolysin from Clostridium perfringens. Eur. J. Biochem. 1987, 167, 425-430.

47. Soltani, C.E.; Hotze, E.M.; Johnson, A.E.; Tweten, R.K. Structural elements of the cholesterol-dependent cytolysins that are responsible for their cholesterol-sensitive membrane interactions. Proc. Natl. Acad. Sci. USA 2007, 104, 20226-20231.

48. Iwamoto, M.; Ohno-Iwashita, Y.; Ando, S. Effect of isolated $C$-terminal fragment of $\theta$-toxin (perfringolysin O) on toxin assembly and membrane lysis. Eur. J. Biochem. 1990, 194, 25-31.

49. Dowd, K.J.; Tweten, R.K. The cholesterol-dependent cytolysin signature motif: A critical element in the allosteric pathway that couples membrane binding to pore assembly. PLoS Pathog. 2012, 8 , e1002787. 
50. Nakamura, M.; Sekino-Suzuki, N.; Mitsui, K.; Ohno-Iwashita, Y. Contribution of tryptophan residues to the structural changes in perfringolysin $\mathrm{O}$ during interaction with liposomal membranes. J. Biochem. 1998, 123, 1145-1155.

51. Farrand, A.J.; LaChapelle, S.; Hotze, E.M.; Johnson, A.E.; Tweten, R.K. Only two amino acids are essential for cytolytic toxin recognition of cholesterol at the membrane surface. Proc. Natl. Acad. Sci. USA 2010, 107, 4341-4346.

52. Ramachandran, R.; Tweten, R.K.; Johnson, A.E. The domains of a cholesterol-dependent cytolysin undergo a major FRET-detected rearrangement during pore formation. Proc. Natl. Acad. Sci. USA 2005, 102, 7139-7144.

53. LaChapelle, S.; Tweten, R.K.; Hotze, E.M. Intermedilysin-receptor interactions during assembly of the pore complex: Assembly intermediates increase host cell susceptibility to complement-mediated lysis. J. Biol. Chem. 2009, 284, 12719-12726.

54. Ohno-Iwashita, Y.; Shimada, Y.; Waheed, A.A.; Hayashi, M.; Inomata, M.; Nakamura, M.; Maruya, M.; Iwashita, S. Perfringolysin O, a cholesterol-binding cytolysin, as a probe for lipid rafts. Anaerobe 2004, 10, 125-134.

55. Ohno-Iwashita, Y.; Iwamoto, M.; Ando, S.; Iwashita, S. Effect of lipidic factors on membrane cholesterol topology-Mode of binding of $\theta$-toxin to cholesterol in liposomes. Biochim. Biophys. Acta 1992, 1109, 81-90.

56. Shimada, Y.; Maruya, M.; Iwashita, S.; Ohno-Iwashita, Y. The $C$-terminal domain of perfringolysin $\mathrm{O}$ is an essential cholesterol-binding unit targeting to cholesterol-rich microdomains. Eur. J. Biochem. 2002, 269, 6195-6203.

57. Waheed, A.A.; Shimada, Y.; Heijnen, H.F.; Nakamura, M.; Inomata, M.; Hayashi, M.; Iwashita, S.; Slot, J.W.; Ohno-Iwashita, Y. Selective binding of perfringolysin O derivative to cholesterol-rich membrane microdomains (rafts). Proc. Natl. Acad. Sci. USA 2001, 98, 4926-4931.

58. Nelson, L.D.; Johnson, A.E.; London, E. How interaction of perfringolysin O with membranes is controlled by sterol structure, lipid structure, and physiological low $\mathrm{pH}$ : Insights into the origin of perfringolysin O-lipid raft interaction. J. Biol. Chem. 2008, 283, 4632-4642.

59. Nelson, L.D.; Chiantia, S.; London, E. Perfringolysin O association with ordered lipid domains: Implications for transmembrane protein raft affinity. Biophys. J. 2010, 99, 3255-3263.

60. Lin, Q.; Wang, T.; Li, H.; London, E. Decreasing Transmembrane Segment Length Greatly Decreases Perfringolysin O Pore Size. J. Membr. Biol. 2015.

61. Ramachandran, R.; Tweten, R.K.; Johnson, A.E. Membrane-dependent conformational changes initiate cholesterol-dependent cytolysin oligomerization and intersubunit $\beta$-strand alignment. Nat. Struct. Mol. Biol. 2004, 11, 697-705.

62. Sato, T.K.; Tweten, R.K.; Johnson, A.E. Disulfide-bond scanning reveals assembly state and $\beta$-strand tilt angle of the PFO $\beta$-barrel. Nat. Chem. Biol. 2013, 9, 383-389.

63. Hotze, E.M.; Wilson-Kubalek, E.; Farrand, A.J.; Bentsen, L.; Parker, M.W.; Johnson, A.E.; Tweten, R.K. Monomer-monomer interactions propagate structural transitions necessary for pore formation by the cholesterol-dependent cytolysins. J. Biol. Chem. 2012, 287, 24534-24543.

64. Rossjohn, J.; Polekhina, G.; Feil, S.C.; Morton, C.J.; Tweten, R.K.; Parker, M.W. Structures of perfringolysin $\mathrm{O}$ suggest a pathway for activation of cholesterol-dependent cytolysins. J. Mol. Biol. 2007, 367, 1227-1236. 
65. Shatursky, O.; Heuck, A.P.; Shepard, L.A.; Rossjohn, J.; Parker, M.W.; Johnson, A.E.; Tweten, R.K. The mechanism of membrane insertion for a cholesterol-dependent cytolysin: A novel paradigm for pore-forming toxins. Cell 1999, 99, 293-299.

66. Shepard, L.A.; Shatursky, O.; Johnson, A.E.; Tweten, R.K. The mechanism of pore assembly for a cholesterol-dependent cytolysin: Formation of a large prepore complex precedes the insertion of the transmembrane $\beta$-hairpins. Biochemistry 2000, 39, 10284-10293.

67. Shepard, L.A.; Heuck, A.P.; Hamman, B.D.; Rossjohn, J.; Parker, M.W.; Ryan, K.R.; Johnson, A.E.; Tweten, R.K. Identification of a membrane-spanning domain of the thiol-activated pore-forming toxin Clostridium perfringens perfringolysin O: An $\alpha$-helical to $\beta$-sheet transition identified by fluorescence spectroscopy. Biochemistry 1998, 37, 14563-14574.

68. Hotze, E.M.; Heuck, A.P.; Czajkowsky, D.M.; Shao, Z.; Johnson, A.E.; Tweten, R.K. Monomer-monomer interactions drive the prepore to pore conversion of a $\beta$-barrel-forming cholesterol-dependent cytolysin. J. Biol. Chem. 2002, 277, 11597-11605.

69. Tweten, R.K. Cholesterol-dependent cytolysins, a family of versatile pore-forming toxins. Infect. Immun. 2005, 73, 6199-6209.

70. White, S.H.; Wimley, W.C. Membrane protein folding and stability: Physical principles. Annu. Rev. Biophys. Biomol. Struct. 1999, 28, 319-365.

71. Kacprzyk-Stokowiec, A.; Kulma, M.; Traczyk, G.; Kwiatkowska, K.; Sobota, A.; Dadlez, M. Crucial role of perfringolysin O D1 domain in orchestrating structural transitions leading to membrane-perforating pores: A hydrogen-deuterium exchange study. J. Biol. Chem. 2014, 289, 28738-28752.

72. Wade, K.R.; Hotze, E.M.; Kuiper, M.J.; Morton, C.J.; Parker, M.W.; Tweten, R.K. An intermolecular electrostatic interaction controls the prepore-to-pore transition in a cholesterol-dependent cytolysin. Proc. Natl. Acad. Sci. USA 2015, 112, 2204-2209.

73. Tilley, S.J.; Orlova, E.V.; Gilbert, R.J.; Andrew, P.W.; Saibil, H.R. Structural basis of pore formation by the bacterial toxin pneumolysin. Cell 2005, 121, 247-256.

74. Wuster, A.; Babu, M.M. Conservation and evolutionary dynamics of the agr cell-to-cell communication system across firmicutes. J. Bacteriol. 2008, 190, 743-746.

75. Ohtani, K.; Yuan, Y.; Hassan, S.; Wang, R.; Wang, Y.; Shimizu, T. Virulence gene regulation by the agr system in Clostridium perfringens. J. Bacteriol. 2009, 191, 3919-3927.

76. Vidal, J.E.; Chen, J.; Li, J.; McClane, B.A. Use of an EZ-Tn5-based random mutagenesis system to identify a novel toxin regulatory locus in Clostridium perfringens strain 13. PLoS ONE 2009, 4, e6232.

77. Schauder, S.; Shokat, K.; Surette, M.G.; Bassler, B.L. The LuxS family of bacterial autoinducers: Biosynthesis of a novel quorum-sensing signal molecule. Mol. Microbiol. 2001, 41, 463-476.

78. Ba-Thein, W.; Lyristis, M.; Ohtani, K.; Nisbet, I.T.; Hayashi, H.; Rood, J.I.; Shimizu, T. The virR/virS locus regulates the transcription of genes encoding extracellular toxin production in Clostridium perfringens. J. Bacteriol. 1996, 178, 2514-2520.

79. Lyristis, M.; Bryant, A.E.; Sloan, J.; Awad, M.M.; Nisbet, I.T.; Stevens, D.L.; Rood, J.I. Identification and molecular analysis of a locus that regulates extracellular toxin production in Clostridium perfringens. Mol. Microbiol. 1994, 12, 761-777. 
80. Okumura, K.; Ohtani, K.; Hayashi, H.; Shimizu, T. Characterization of genes regulated directly by the VirR/VirS system in Clostridium perfringens. J. Bacteriol. 2008, 190, 7719-7727.

81. Shimizu, T.; Ba-Thein, W.; Tamaki, M.; Hayashi, H. The virR gene, a member of a class of two-component response regulators, regulates the production of perfringolysin $\mathrm{O}$, collagenase, and hemagglutinin in Clostridium perfringens. J. Bacteriol. 1994, 176, 1616-1623.

82. Ohtani, K.; Hayashi, H.; Shimizu, T. The luxS gene is involved in cell-cell signalling for toxin production in Clostridium perfringens. Mol. Microbiol. 2002, 44, 171-179.

83. Cheung, J.K.; Awad, M.M.; McGowan, S.; Rood, J.I. Functional analysis of the VirSR phosphorelay from Clostridium perfringens. PLoS ONE 2009, 4, e5849.

84. Cheung, J.K.; Rood, J.I. The VirR response regulator from Clostridium perfringens binds independently to two imperfect direct repeats located upstream of the pfoA promoter. J. Bacteriol. 2000, 182, 57-66.

85. Cheung, J.K.; Dupuy, B.; Deveson, D.S.; Rood, J.I. The spatial organization of the VirR boxes is critical for VirR-mediated expression of the perfringolysin $\mathrm{O}$ gene, $p f o A$, from Clostridium perfringens. J. Bacteriol. 2004, 186, 3321-3330.

86. Awad, M.M.; Rood, J.I. Perfringolysin O expression in Clostridium perfringens is independent of the upstream $p f o R$ gene. J. Bacteriol. 2002, 184, 2034-2038.

87. Dworkin, M.; Falkow, S. The Prokaryotes: A Handbook on the Biology of Bacteria, 3rd ed.; Springer: New York, NY, USA, 2006; pp. 698-770.

88. Titball, R.W. Gas gangrene: An open and closed case. Microbiology 2005, 151, 2821-2828.

89. Bryant, A.E.; Stevens, D.L. Clostridial myonecrosis: New insights in pathogenesis and management. Curr. Infect. Dis. Rep. 2010, 12, 383-391.

90. Beran, G.W. Handbook of Zoonoses, 2nd ed.; CRC Press: Boca Raton, FL, USA, 1994; pp. 127-138.

91. Ninomiya, M.; Matsushita, O.; Minami, J.; Sakamoto, H.; Nakano, M.; Okabe, A. Role of $\alpha$-toxin in Clostridium perfringens infection determined by using recombinants of $C$. perfringens and Bacillus subtilis. Infect. Immun. 1994, 62, 5032-5039.

92. Awad, M.M.; Bryant, A.E.; Stevens, D.L.; Rood, J.I. Virulence studies on chromosomal $\alpha$-toxin and $\theta$-toxin mutants constructed by allelic exchange provide genetic evidence for the essential role of $\alpha$-toxin in Clostridium perfringens-mediated gas gangrene. Mol. Microbiol. 1995, 15, 191-202.

93. Ellemor, D.M.; Baird, R.N.; Awad, M.M.; Boyd, R.L.; Rood, J.I.; Emmins, J.J. Use of genetically manipulated strains of Clostridium perfringens reveals that both $\alpha$-toxin and $\theta$-toxin are required for vascular leukostasis to occur in experimental gas gangrene. Infect. Immun. 1999, 67, 4902-4907.

94. Stevens, D.L.; Tweten, R.K.; Awad, M.M.; Rood, J.I.; Bryant, A.E. Clostridial gas gangrene: Evidence that $\alpha$ and $\theta$ toxins differentially modulate the immune response and induce acute tissue necrosis. J. Infect. Dis. 1997, 176, 189-195.

95. Stevens, D.L.; Bryant, A.E. Pathogenesis of Clostridium perfringens infection: Mechanisms and mediators of shock. Clin. Infect. Dis. 1997, 25 (Suppl. 2), S160-S164.

96. Asmuth, D.M.; Olson, R.D.; Hackett, S.P.; Bryant, A.E.; Tweten, R.K.; Tso, J.Y.; Zollman, T.; Stevens, D.L. Effects of Clostridium perfringens recombinant and crude phospholipase $\mathrm{C}$ and $\theta$-toxin on rabbit hemodynamic parameters. J. Infect. Dis. 1995, 172, 1317-1323. 
97. Bryant, A.E.; Bergstrom, R.; Zimmerman, G.A.; Salyer, J.L.; Hill, H.R.; Tweten, R.K.; Sato, H.; Stevens, D.L. Clostridium perfringens invasiveness is enhanced by effects of $\theta$ toxin upon PMNL structure and function: The roles of leukocytotoxicity and expression of CD11/CD18 adherence glycoprotein. FEMS Immunol. Med. Microbiol. 1993, 7, 321-336.

98. Bryant, A.E.; Chen, R.Y.; Nagata, Y.; Wang, Y.; Lee, C.H.; Finegold, S.; Guth, P.H.; Stevens, D.L. Clostridial gas gangrene. I. Cellular and molecular mechanisms of microvascular dysfunction induced by exotoxins of Clostridium perfringens. J. Infect. Dis. 2000, 182, 799-807.

99. Hickey, M.J.; Kwan, R.Y.; Awad, M.M.; Kennedy, C.L.; Young, L.F.; Hall, P.; Cordner, L.M.; Lyras, D.; Emmins, J.J.; Rood, J.I. Molecular and cellular basis of microvascular perfusion deficits induced by Clostridium perfringens and Clostridium septicum. PLoS Pathog. 2008, 4, e1000045.

100. Bryant, A.E.; Chen, R.Y.; Nagata, Y.; Wang, Y.; Lee, C.H.; Finegold, S.; Guth, P.H.; Stevens, D.L. Clostridial gas gangrene. II. Phospholipase C-induced activation of platelet gpIIbIIIa mediates vascular occlusion and myonecrosis in Clostridium perfringens gas gangrene. J. Infect. Dis. 2000, 182, 808-815.

101. Bryant, A.E.; Bayer, C.R.; Hayes-Schroer, S.M.; Stevens, D.L. Activation of platelet gpIIbIIIa by phospholipase $\mathrm{C}$ from Clostridium perfringens involves store-operated calcium entry. J. Infect. Dis. 2003, 187, 408-417.

102. Bryant, A.E.; Bayer, C.R.; Aldape, M.J.; Wallace, R.J.; Titball, R.W.; Stevens, D.L. Clostridium perfringens phospholipase C-induced platelet/leukocyte interactions impede neutrophil diapedesis. J. Med. Microbiol. 2006, 55, 495-504.

103. Stevens, D.L.; Troyer, B.E.; Merrick, D.T.; Mitten, J.E.; Olson, R.D. Lethal effects and cardiovascular effects of purified $\alpha$ - and $\theta$-toxins from Clostridium perfringens. J. Infect. Dis. 1988, 157, 272-279.

104. Bryant, A.E.; Stevens, D.L. Phospholipase C and perfringolysin O from Clostridium perfringens upregulate endothelial cell-leukocyte adherence molecule 1 and intercellular leukocyte adherence molecule 1 expression and induce interleukin-8 synthesis in cultured human umbilical vein endothelial cells. Infect. Immun. 1996, 64, 358-362.

105. Whatley, R.E.; Nelson, P.; Zimmerman, G.A.; Stevens, D.L.; Parker, C.J.; McIntyre, T.M.; Prescott, S.M. The regulation of platelet-activating factor production in endothelial cells. The role of calcium and protein kinase C. J. Biol. Chem. 1989, 264, 6325-6333.

106. Bunting, M.; Lorant, D.E.; Bryant, A.E.; Zimmerman, G.A.; McIntyre, T.M.; Stevens, D.L.; Prescott, S.M. a toxin from Clostridium perfringens induces proinflammatory changes in endothelial cells. J. Clin. Invest. 1997, 100, 565-574.

107. Stevens, D.L.; Bryant, A.E. The role of clostridial toxins in the pathogenesis of gas gangrene. Clin. Infect. Dis. 2002, 35, S93-S100.

108. Stevens, D.L.; Mitten, J.; Henry, C. Effects of $\alpha$ and $\theta$ toxins from Clostridium perfringens on human polymorphonuclear leukocytes. J. Infect. Dis. 1987, 156, 324-333.

109. O'Brien, D.K.; Melville, S.B. The anaerobic pathogen Clostridium perfringens can escape the phagosome of macrophages under aerobic conditions. Cell. Microbiol. 2000, 2, 505-519.

110. O’Brien, D.K.; Melville, S.B. Effects of Clostridium perfringens $\alpha$-toxin (PLC) and perfringolysin $\mathrm{O}$ (PFO) on cytotoxicity to macrophages, on escape from the phagosomes of macrophages, and on persistence of $C$. perfringens in host tissues. Infect. Immun. 2004, 72, 5204-5215. 
111. Sayeed, S.; Uzal, F.A.; Fisher, D.J.; Saputo, J.; Vidal, J.E.; Chen, Y.; Gupta, P.; Rood, J.I.; McClane, B.A. $\beta$ toxin is essential for the intestinal virulence of Clostridium perfringens type $\mathrm{C}$ disease isolate CN3685 in a rabbit ileal loop model. Mol. Microbiol. 2008, 67, 15-30.

112. Valgaeren, B.R.; Pardon, B.; Verherstraeten, S.; Goossens, E.; Timbermont, L.; Haesebrouck, F.; Ducatelle, R.; Deprez, P.R.; van Immerseel, F. Intestinal clostridial counts have no diagnostic value in the diagnosis of enterotoxaemia in veal calves. Vet. Rec. 2013, 172, 237.

113. Manteca, C.; Daube, G.; Pirson, V.; Limbourg, B.; Kaeckenbeeck, A.; Mainil, J.G. Bacterial intestinal flora associated with enterotoxaemia in Belgian Blue calves. Vet. Microbiol. 2001, 81, 21-32.

114. Muylaert, A.; Lebrun, M.; Duprez, J.N.; Labrozzo, S.; Theys, H.; Taminiau, B.; Mainil, J. Enterotoxaemia-like syndrome and Clostridium perfringens in veal calves. Vet. Rec. 2010, 167, $64-65$.

115. Pardon, B.; De Bleecker, K.; Hostens, M.; Callens, J.; Dewulf, J.; Deprez, P. Longitudinal study on morbidity and mortality in white veal calves in Belgium. BMC Vet. Res. 2012, 8, 26.

116. Lebrun, M.; Mainil, J.G.; Linden, A. Cattle enterotoxaemia and Clostridium perfringens: Description, diagnosis and prophylaxis. Vet. Rec. 2010, 167, 13-22.

117. Morris, W.E.; Venzano, A.J.; Elizondo, A.; Vilte, D.A.; Mercado, E.C.; Fernandez-Miyakawa, M.E. Necrotic enteritis in young calves. J. Vet. Diagn. Invest. 2011, 23, 254-259.

118. Valgaeren, B.; Pardon, B.; Goossens, E.; Verherstraeten, S.; Schauvliege, S.; Timbermont, L.; Ducatelle, R.; Deprez, P.; van Immerseel, F. Lesion development in a new intestinal loop model indicates the involvement of a shared Clostridium perfringens virulence factor in haemorrhagic enteritis in calves. J. Comp. Pathol. 2013, 149, 103-112.

119. Sakurai, J.; Nomura, S.; Fujii, Y.; Oshita, Y. Effect of Clostridium perfringens $\alpha$ toxin on the isolated rat vas deferens. Toxicon 1985, 23, 449-455.

120. Uzal, F.A.; Songer, J.G. Diagnosis of Clostridium perfringens intestinal infections in sheep and goats. J. Vet. Diagn. Invest. 2008, 20, 253-265.

121. Sayeed, S.; Fernandez-Miyakawa, M.E.; Fisher, D.J.; Adams, V.; Poon, R.; Rood, J.I.; Uzal, F.A.; McClane, B.A. $\varepsilon$-toxin is required for most Clostridium perfringens type D vegetative culture supernatants to cause lethality in the mouse intravenous injection model. Infect. Immun. 2005, 73, 7413-7421.

122. Garcia, J.P.; Adams, V.; Beingesser, J.; Hughes, M.L.; Poon, R.; Lyras, D.; Hill, A.; McClane, B.A.; Rood, J.I.; Uzal, F.A. $\varepsilon$ toxin is essential for the virulence of Clostridium perfringens type D infection in sheep, goats, and mice. Infect. Immun. 2013, 81, 2405-2414.

123. Shewell, L.K.; Harvey, R.M.; Higgins, M.A.; Day, C.J.; Hartley-Tassell, L.E.; Chen, A.Y.; Gillen, C.M.; James, D.B.; Alonzo, F., 3rd; Torres, V.J.; et al. The cholesterol-dependent cytolysins pneumolysin and streptolysin $\mathrm{O}$ require binding to red blood cell glycans for hemolytic activity. Proc. Natl. Acad. Sci. USA 2014, 111, E5312-E5320.

(C) 2015 by the authors; licensee MDPI, Basel, Switzerland. This article is an open access article distributed under the terms and conditions of the Creative Commons Attribution license (http://creativecommons.org/licenses/by/4.0/). 